\title{
Oxidative Stress and Antioxidant Markers in Serum and Red Blood Cell of Patients with Ischemic and Hemorrhagic Stroke: A Case-Control Study
}

\author{
Maryam Valavi, ${ }^{1}$ Asghar Zarban,, ${ }^{,}$Mohammad Dehghani Firuzabadi, ${ }^{2}$ Gholamreza Sharifzadeh, ${ }^{3}$ and \\ Masume Zarezade ${ }^{4}$ \\ ${ }^{1}$ Department of Clinical Biochemistry, Faculty of Medicine, Birjand University of Medical Sciences, Birjand, IR Iran \\ ${ }^{2}$ Department of Neurology, Faculty of Medicine, Birjand University of Medical Sciences, Birjand, IR Iran \\ ${ }^{3}$ Social Determinants of Health Research Center, Birjand University of Medical Sciences, Birjand, Iran \\ ${ }^{4}$ Department of Clinical Biochemistry, Faculty of Medicine, Birjand University of Medical Sciences, Birjand, IR Iran \\ "Corresponding author: Asghar Zarban, Associate Professor, Department of Clinical Biochemistry, Faculty of Medicine, Birjand University of Medical Sciences, Ghaffari Street, \\ Birjand, IR Iran. Tel: +98-5632395000, E-mail: azarban@yahoo.com
}

Received 2016 March 22; Revised 2016 April 29; Accepted 2016 May 04.

\begin{abstract}
Background and Aim: Oxidative stress is a major mechanism involved in stroke pathogenesis. The present study evaluated and compared oxidative stress parameters in patients with ischemic and hemorrhagic stroke (IS and HS) at the time of hospitalization and 1 week after hospitalization.

Methods: This was a case-control study with 87 stroke patients (58 IS and 29 HS) as the case and 58 normal individuals as the control group. Sampling was done at the time of hospitalization and after 1 week. The serum total antioxidant capacity (TAC), thiol group, and F2-isoprostan levels were measured using Ferric Reducing Antioxidant Power (FRAP), Elman, and Enzyme Linked Immunosorbent Assay ELISA methods, respectively.

Results: At the time of hospitalization, there was no significant difference in TAC and F2-isoprostan levels between the case and control groups (TAC for IS $=852.4 \pm 238.9, \mathrm{HS}=1064.1 \pm 271.1$, and control $=947.6 \pm 203.1 \mu \mathrm{mol} / \mathrm{L} ; \mathrm{P}=0.074$ ), yet, the thiol group levels were significantly lower $(\mathrm{P}<0.001)$ in the case groups (IS and HS) compared to the control group (IS $=360.3 \pm 139.8$, HS $=$ $357.8 \pm 94.4$, and control $=568.4 \pm 201.4 \mathrm{mmol} / \mathrm{L} ; \mathrm{P}<0.001)$. At 1 week after the time of hospitalization, the case patients showed a significant increase in thiol levels (IS at $\mathrm{p}=0.005$; HS at $\mathrm{P}=0.02)$, yet, TAC values decreased $(\mathrm{P}<0.015)$ only in patients with HS compared to levels at the time of hospitalization. There were no significant differences between groups for antioxidant enzymes (SOD, GPx, and CAT) or the National institute of health stroke scale (NIHSS) score of patients at the time of hospitalization and 1 week later, except for superoxide dismutase (SOD), which increased in patients with $\mathrm{HS}(\mathrm{P}=0.05)$.

Conclusions: The results showed that antioxidant defense status in stroke patients was lower than for the control group. Improving the antioxidant defense system, especially for HS patients by strategies such as natural antioxidant consumption is suggested.
\end{abstract}

Keywords: Ischemic, Hemorrhagic, Oxidative Stress, Thiol, Total Antioxidant Capacity, Antioxidant Enzyme, NIHSS

\section{Background}

Stroke or cerebrovascular accident is the third leading cause of death in the United States and the most common disabling neurological disease in adults in most places (1, 2 ). This disorder is caused by interruption of blood flow to a particular area of the brain. If part of the brain is deprived of adequate blood supply, brain cells will become damaged or die $(2,3)$. Strokes are divided pathologically to ischemic and hemorrhagic types. Ischemic stroke (IS) accounts for $87 \%$ of strokes and occurs when a blood clot or clump of fat occludes blood flow to the brain. Hemorrhagic stroke (HS) accounts for only 13\% of strokes and results from rupture of a blood vessel within or around the brain $(2,4)$. Recent identification of several molecules in- volved in the death of neurons have proven promising in the identification of the pathogenesis of brain damage following both ischemic and hemorrhagic strokes.

Oxidative stress is a mechanism involved in nerve damage caused by stroke (5-8) and is the result of an imbalance between the production of free radicals (reactive oxygen species) and the antioxidant defense $\operatorname{system}(1,9)$. This phenomenon leads to cellular damage, cell death and acceleration of degenerative diseases associated with aging such as cancer, cardiovascular disease, diabetes, and pulmonary and neural degenerative diseases $(9,10)$. Increased production of free radicals and other chemical species has been confirmed both in ischemic and hemorrhagic strokes, and oxidative stress was introduced as a fundamental mecha-

Copyright ( ) 2016, Modern Care Journal. This is an open-access article distributed under the terms of the Creative Commons Attribution-NonCommercial 4.0 International License (http://creativecommons.org/licenses/by-nc/4.0/) which permits copy and redistribute the material just in noncommercial usages, provided the original work is properly cited. 
nism in brain damage under these conditions (1).

Free radicals change the structure and function of target molecules by taking their electrons. Oxidants also effect cell membranes and genetic material such as DNA and RNA, and various enzymatic events, causing cell damage during ischemia and reperfusion $(4,9,11)$. Despite this, a few studies have examined the effects of oxidative stress on hemorrhagic stroke (4). The current study evaluated oxidative stress parameters in the serum and red blood cell of patients experiencing ischemic and hemorrhagic strokes at the time of hospitalization and compared them with the values of these patients 1 week later.

\section{Methods}

This case-control study was conducted on an overall population of 135 individuals (58 IS and $29 \mathrm{HS}$ as the case groups; 58 healthy individuals as the control group). After diagnosis and hospitalization of the patients at Valiasr hospital as well as confirmation of stroke type by a specialist, blood samples were taken both at the time of hospitalization and 1 week later. Having a background of stroke or heart attack, pulmonary, liver, kidney disease, and cancers was considered as the exclusion criteria. The control group was chosen from the healthy population, which matched for age and gender with the same exclusion criteria. Blood samples were obtained from the controls at the given time spans.

Serum samples and hemolysis from red blood cells after preparation were stored at $-80^{\circ} \mathrm{C}$ until testing. The serum total antioxidant capacity (TAC) was measured by ferric reducing/antioxidant power (FRAP) (12), serum F2 isoprostane level was measured by Enzyme Linked Immunosorbent Assay (ELISA) using a ZellBio kit (Germany), and thiol levels were determined using Ellman's method (13). The enzyme activity of Superoxide Dismutase (SOD) was determined using the Marklund and Marklund method (14), Catalase (CAT) using Abei's method (15) and Glutathione peroxidase (GPx) using Paglia's method (16). Hemolysate prepared from red blood cells was measured and their activity was calculated relative to the amount of hemoglobin. The NIHSS scale was used to evaluate the effect of acute stroke. The collected data was then analyzed using the SPSS 19 software with the paired t test, Analysis of Variance(ANOVA), Kruskal-Wallis, Tukey's test, Wilcoxon, one-way repeated ANOVA, and chi square test at $\alpha=0.05$ significance level.

\section{Results}

The average age of the patients in the IS, HS, control group was $69.4 \pm 13.8,67.1 \pm 16.5$, and $70.2 \pm 12.5$, respec- tively. Regarding gender there were 27 males (46.6\%) in the IS group, 14 (48.6\%) in the HS group, and 28 in the control group (48.3\%). The remaining subjects were female. All groups were similar regarding age and gender $(\mathrm{P}>0.05)$. On the first day of the study, the mean serum TAC level showed no significant differences when comparing the IS and HS groups with the control group, although it was significantly higher in the HS group than in the IS group (1064.1 versus 854.2).

The mean thiol index was significantly lower in the IS and HS groups than in the control group. There was no significant difference in the isoprostane index between groups (Table 1). After one week, the thiol index increased in the 2 groups compared to the first day of hospitalization, yet, the serum TAC index was significantly lower in the HS group. No significant difference was observed in the isoprostane index between the first and seventh day in the 2 stroke groups (Table 2).

There were no significant differences in antioxidant enzymes activity (relative to hemoglobin) in red blood cells (RBCs) between the different groups (Table 3). The SOD level in the HS group increased from the first to seventh day (Table 4). The mean NIHSS index in the IS and HS group was $7.11 \pm 7.63$ and $9.93 \pm 8.92$, respectively $(\mathrm{P}=0.18$; $\mathrm{Z}$ $=1.33$ ). This was no significant difference between the 2 stroke groups.

\section{Discussion}

The TAC analysis showed no significant difference between the stroke and control groups, although the TAC value for the HS group was significantly greater than that of the IS group $(\mathrm{P}<0.001)$. Studies have found that TAC decreases more in post-acute stroke patients when compared to healthy controls $(17,18)$. The current similarly found that TAC was lower in IS patients than in controls $(\mathrm{P}=0.074)$. In the HS group, TAC levels decreased significantly by the seventh day $(P=0.015)$. Icme et al. (4) and Sheikh et al. (19) investigated oxidative stress parameters in HS and IS patients. Both reported no significant differences between stroke groups and healthy controls, although their values were lower in the case groups than in the control group.

The current findings are in contrast with those from Erel (20) concerning the HS group. They found that TAC in the HS group decreased significantly more than the control group. They used the Erel method to measure TAC and found that a defective system of free radicals may increase intracerebral hemorrhagic oxidative damage (21). The difference in TAC measurement methods could be the reason for the inconsistent results between the current study and that of Genula at al. and Ritzenthler et al. (22), which found 
Table 1. Comparison of the Mean Serum Antioxidant Status, Thiol and F2 Isoprostane in the Three Groups on the First Day ${ }^{\mathrm{a}, \mathrm{b}}$

\begin{tabular}{|c|c|c|c|c|c|}
\hline \multirow[t]{2}{*}{ Variable name } & \multicolumn{4}{|c|}{ Group Under Study } & \multirow[t]{2}{*}{ ANOVA/Tukey's Test } \\
\hline & Ischemic $(\mathrm{N}=58)$ & Hemorrhagic $(N=29)$ & \multicolumn{2}{|c|}{ Control $(\mathrm{N}=58)$} & \\
\hline Total Antioxidant capacity, $\mu \mathrm{mol} / \mathrm{L}$ & $852.4 \pm 238.9$ & $1064.1 \pm 271.1$ & \multicolumn{2}{|c|}{$947.6 \pm 203.1$} & $<0.001$ \\
\hline F2 isoprostane, pg/mL & $271 / 8 \pm 151 / 3$ & $307 / 7 \pm 183 / 8$ & \multicolumn{2}{|c|}{$330 / 0 \pm 153 / 8$} & 0.29 \\
\hline \multicolumn{6}{|l|}{$\begin{array}{l}{ }^{\mathrm{a}} \text { Values are expressed as mean } \pm \text { SD. } \\
{ }^{\mathrm{b}} \alpha=0.05 . \\
{ }^{\mathrm{c}} \text { Ischemic. } \\
{ }^{\mathrm{d}} \text { Hemorrhagic. }\end{array}$} \\
\hline \multicolumn{6}{|c|}{ able 2. Comparison of the Mean Serum Thiol Index and Total Antioxidant Capacity and F2isoprostane on the First and Seventh Day After Stroke in The Two Case Groups ${ }^{\mathrm{a}}$} \\
\hline \multirow[t]{2}{*}{ Group Under Study } & & & \multicolumn{2}{|c|}{ Time } & P Value Paired T-Test \\
\hline & & & 1st Day & 7th Day & \\
\hline \multirow{2}{*}{ Thiol, mmol/L } & & Ischemic $(\mathrm{N}=58)$ & $357.8 \pm 139.9$ & $470.1 \pm 247.1$ & $0.005^{\mathrm{b}}$ \\
\hline & & Hemorrhagic $(\mathrm{N}=29)$ & $357.7 \pm 94.4$ & $439.2 \pm 159.5$ & $0.02^{\mathrm{b}}$ \\
\hline \multirow{2}{*}{ Total antioxidant capacity, $\mu \mathrm{mol} / \mathbf{L}$} & & Ischemic $(\mathrm{N}=58)$ & $852.4 \pm 238.9$ & $884.4 \pm 227.4$ & 0.410 \\
\hline & & Hemorrhagic $(\mathrm{N}=29)$ & $1064.1 \pm 271.1$ & $920.1 \pm 306.9$ & $0.015^{\mathrm{b}}$ \\
\hline \multirow{2}{*}{ F2 isoprostane, pg/mL } & & Ischemic $(\mathrm{N}=58)$ & $271.8 \pm 151.3$ & $310.4 \pm 171.5$ & 0.09 \\
\hline & & Hemorrhagic $(\mathrm{N}=29)$ & $307.7 \pm 183.8$ & $298.8 \pm 157.3$ & 0.66 \\
\hline
\end{tabular}

${ }^{\mathrm{a}}$ Values are expressed as mean $\pm \mathrm{SD}$.

${ }^{\mathrm{b}} \alpha=0.05$.

Table 3. Comparison of the Mean Enzymes Activity of SOD/HGB, GPX/HGB and CAT/HGB in the Three Groups on the First Day

\begin{tabular}{lcccc}
\hline Variable Name & \multicolumn{2}{c}{ Group Under Study } & \multicolumn{1}{c}{ Anova or Kruskalwallis Test } \\
\cline { 2 - 4 } & Ischemic $(\mathbf{N}=\mathbf{5 8})$ & Hemorrhagic $(\mathbf{N}=\mathbf{2 9})$ & Control $(\mathbf{N}=\mathbf{5 8})$ \\
\hline SOD/HGB, IU/L & $4.72 \pm 5.18$ & $4.28 \pm 3.1$ & $3.21 \pm 2.69$ & 0.36 \\
GPX/HGB, IU/L & $12.67 \pm 3.5$ & $13.61 \pm 4.6$ & $14.93 \pm 5.6$ & 0.11 \\
CAT/HGB, (IU/L & $174.6 \pm 55.4$ & $182.4 \pm 63.2$ & $181.6 \pm 78.2$ \\
\hline
\end{tabular}

${ }^{\mathrm{a}}$ Values are expressed as mean $\pm \mathrm{SD}$.

Table 4. Comparison of the Mean Enzymes Activity of SOD/HGB, GPX/HGB and CAT/HGB in Three Groups on the First and Seventh Day After Stroke in the Two Case Groups

\begin{tabular}{|c|c|c|c|c|}
\hline \multirow[t]{2}{*}{ Group Under Study } & & \multicolumn{2}{|c|}{ Time } & \multirow[t]{2}{*}{ P Value Paired T-Test } \\
\hline & & 1st Day & 7th Day & \\
\hline SOD/HGB, IU/L & Ischemic $(\mathrm{N}=58)$ & $4.72 \pm 5.18$ & $4.43 \pm 5.64$ & 0.75 \\
\hline \multirow{2}{*}{ GPX/HGB, IU/L } & Ischemic $(\mathrm{N}=58)$ & $12.67 \pm 3.5$ & $13.65 \pm 2.77$ & 0.11 \\
\hline & Hemorrhagic $(\mathrm{N}=29)$ & $13.61 \pm 4.6$ & $14.04 \pm 4.43$ & 0.67 \\
\hline \multirow{2}{*}{ CAT/HGB, IU/L } & Ischemic $(\mathrm{N}=58)$ & $174.6 \pm 55.4$ & $181.6 \pm 65.4$ & 0.39 \\
\hline & Hemorrhagic $(\mathrm{N}=29)$ & $182.4 \pm 63.2$ & $182.6 \pm 50.48$ & 0.66 \\
\hline
\end{tabular}

${ }^{\mathrm{a}}$ Values are expressed as mean $\pm \mathrm{SD}$

${ }^{\mathrm{b}} \alpha=0.05$.

that TAC decreased on the first day following IS and continued to decrease for nearly 5 days.

Studies have compared oxidative damage in HS and IS patients and healthy controls (4). Parizadeh et al. reported that oxidative parameters are not useful predictors and evaluators of trends during the first 6 months after stroke (23). Several studies have stated that the increase in TAC after stroke may protect the victim from the harmful effects 
of free radicals produced during ischemia or upon revascularization $(6,17)$. Nanetti et al. (7) also found that medicine can increase TAC in patients during the first month.

The thiol index decreased significantly more in HS and IS patients than in the controls $(\mathrm{P}<0.001)$. There was no significant difference between the HS and IS groups in the current study as well as in the study of Icme et al. (4). In the current study, the thiol index increased significantly in HS and IS over the first 7 days. Tsai et al. (24) reported that the amount of free thiols in acute stroke patients increased by the seventh day to a level similar to that of the control group. Several studies reported that the thiol level in IS patients was significantly lower than in the control $(4,24,25)$. Thiols are organic compounds that contain a sulfhydryl ($\mathrm{SH})$ group that protects against oxidative stress.

Bektas et al. (25) proposed that thiol/disulfide hemostat is impaired in IS patients and, as the thiol level decreases any medicinal drug that contains the sulfhydryl group can increase H2S levels. Furthermore, H2S is itself is associated with vasodilatation. As a thiol and a mucolytic and neuroprotective agent, $\mathrm{N}$-Acetylcysteine (NAC) is a precursor of L-cysteine and reduces glutathione. The NAC is a source of sulfhydryl groups in the cell. The antioxidant property of NAC as a sulfhydryl donor may be associated with the regeneration of endothelium-derived relaxing factor and glutathione; thus, if NAC is used to substitute thiol or alpha-lipoic acid or if the thiol-disulfide imbalance is corrected, treatment of IS can be better managed.

In the current study, there was no significant difference between groups in terms of serum F2-isoprostane levels. Beer et al. (26) maintained that oxidative stress reaches its peak on the third day after stroke. They observed a significant increase in F2-isoprostane levels in samples collected in the first phase of stroke (the first 6 hours). There was also a significant difference between the sub-groups of IS in terms of F2-isoprostane levels. F2-isoprostanes, also called 8-epi PGF2 $\alpha$, are of particular interest to researchers because they have platelet vasoconstriction activation properties as well as mitogenic properties (27).

These compounds are of non-enzymatic origin and in eicosanoid family, and are generated by oxygen radicals through random oxidation of tissue phospholipids. Several studies have indicated that isoprostanes are reliable markers for examining oxidative stress under in vivo conditions. Sanchez-Moreno et al. (27) reported that F2isoprostane 8-epiPGF2 $\alpha$ plasma was significantly greater in the stroke group than the control group. They assessed the presence of metabolites derived from prostaglandin, which were formed by random oxidation of tissue phospholipids via oxygen radicals. As markers of oxidative stress, these compounds have the potential to retract ves- sels and swing platelet aggregations.

There is insufficient information about the type of F2isoprostane, time interval between sample collection and centrifugation, and the storage, freezing, and de-freezing temperatures in different studies. Additionally, the isoprostane was measured in either plasma or urine in some studies. In the present study, serum F2-isoprostane was measured. F2-isoprostane disappears from the blood circulation very quickly, thus, its levels indicate continuous and discrete expression over time. Furthermore, sampling, transference, and storage of blood samples for long periods produces additional F2-isoprostane. There are several methods of measuring F2-isoprostane in body liquids, each of which measures a specific isoprostane. Moreover, different kits and methods have been used in different studies. Any of these factors can be a reason for the inconsistency between the current findings and those of other studies (28).

In this study, SOD, GPx, and CAT were investigated. No significant differences were found between groups for these parameters on the first day of hospitalization; however, after 7 days, only the erythrocyte SOD activity in HS patients had increased. The SOD is the main catalyzer of oxygen anions and dismutase them into hydrogen peroxide, thereby inhibiting the accumulation of these free radicals. Hydrogen peroxide is, in turn, trapped by CAT and GPx and converted to $\mathrm{H}_{2} \mathrm{O}$ and $\mathrm{O}_{2}$ molecules. The balance between oxidative and antioxidant mechanisms of these enzymes is a critical component in cells against nerve damage associated with oxidative stress (29). Antioxidant enzymes in the plasma and red blood cells are the most important factors preventing oxidative damage caused by neurological diseases such as stroke (17).

Reports on the effects of SOD activity on acute stroke are contradictory. Similar to the current study, El Kossi et al. (2000) found no significant difference between the IS group and the control group, concerning serum SOD activity (30). On the contrary, Cherubini et al. and Demikaya et al. $(1,31)$ found that SOD activity decreases significantly in IS patients. Cherobini et al. (2000) reported that the levels of CAT, GPx, and SOD activity in plasma and red blood cells in patients at the onset of stroke were lower than the control group. After 1 week, CAT and GPx levels in the patient and control groups were similar. During the same time, however, SOD in the plasma was lower, and in red blood cells was higher than in the controls. The increase in SOD activity after 1 week corresponds with the results of the present study for HS patients (32). Milanlioglu et al. (29) found similar results to that of the present study; no significant differences in serum GPx was found between stroke patients and healthy controls. Demirkaya et al. (2001) found that red blood cell GPx activity decreased in the first 
24 hours after the onset of stroke symptoms as compared with the control group. Aygul et al. (33) also observed that GPx activity in plasma and red blood cells decreased in IS patients.

In the current study, erythrocyte CAT activity of the red blood cells in the stroke groups showed no significant difference with the control group. In this regard, Sheikh et al. (19) reported similar findings. According to some previous reports, the different measurement methods and isoenzymes are the underlying reason for the contradictory behavior; however, further research is needed to investigate these behaviors $(29,32)$.

The NIHSS index was measured to evaluate the effect of acute stroke. No significant differences were found between the IS and HS groups in this regard. This finding is in line with that of Icme et al. (4). The NIHSS index is used to evaluate the effects of acute stroke on consciousness, attention, visual field, eye movement, muscle strength, speech, sensory function, and ataxias. The index is a 15-degree scale based on a neurological examination. Each patient is scored on a 0 to 5 scale where 0 indicates normal, according to his/her responses and movement abilities (34).

\subsection{Conclusions}

This study showed that antioxidant defense status, especially the thiol index, is lower in IS and HS patients than in controls, although oxidative stress indices increased at 1 week post hospitalization. A decrease in oxidative stress and an improved antioxidant defense system, particularly from consumption of natural antioxidants, may reduce the risk of stroke and its complications.

\section{Acknowledgments}

This paper was extracted from a dissertation thesis submitted at Birjand University of Medical Sciences with the research project code 786 and ethics code Ir.bums.1394.9. The authors, hereby appreciate all the patients and healthy individuals, who participated in this study.

\section{References}

1. Cherubini A, Ruggiero C, Polidori MC, Mecocci P. Potential markers of oxidative stress in stroke. Free Radic Biol Med. 2005;39(7):841-52. doi: 10.1016/j.freeradbiomed.2005.06.025. [PubMed: 16140205].

2. Ropper AH. Adams and Victor's principles of neurology. New York: McGraw-Hill Medical Pub; 2005.

3. SOMAY G, TOPALOĞLU P, Somay H, Araal O, Halaç GU, Bulkan M. Cerebrovascular risk factors and stroke subtypes in different age groups: a hospital-based study. TurkJ Med Sci. 2006;36(1):23-9.

4. Icme F, Erel O, Avci A, Satar S, Gulen M, Acehan S. The relation between oxidative stress parameters, ischemic stroke, and hemorrhagic stroke. TurkJ Med Sci. 2015;45(4):947-53. [PubMed: 26422872].
5. Alexandrova ML, Bochev PG. Oxidative stress during the chronic phase after stroke. Free Radic Biol Med. 2005;39(3):297-316. doi: 10.1016/j.freeradbiomed.2005.04.017. [PubMed:15993329].

6. Cojocaru IM, Cojocaru M, Sapira V, Ionescu A. Evaluation of oxidative stress in patients with acute ischemic stroke. Rom J Intern Med. 2013;51(2):97-106. [PubMed: 24294813].

7. Nanetti L, Raffaelli F, Vignini A, Perozzi C, Silvestrini M, Bartolini $\mathrm{M}$, et al. Oxidative stress in ischaemic stroke. Eur J Clin Invest. 2011;41(12):1318-22. doi: 10.1111/j.1365-2362.2011.02546.x. [PubMed: 21623777].

8. Ozkul A, Akyol A, Yenisey C, Arpaci E, Kiylioglu N, Tataroglu C. Oxidative stress in acute ischemic stroke. J Clin Neurosci. 2007;14(11):1062-6. doi: 10.1016/j.jocn.2006.11.008. [PubMed:17884504].

9. Halliwell B, Gutteridge JMC. Free radicals in biology and medicine. USA: Oxford University Press; 2015.

10. Allen CL, Bayraktutan U. Oxidative stress and its role in the pathogenesis of ischaemic stroke. Int J Stroke. 2009;4(6):461-70. doi:10.1111/j.17474949.2009.00387.x. [PubMed:19930058].

11. Valko M, Leibfritz D, Moncol J, Cronin MT, Mazur M, Telser J. Free radicals and antioxidants in normal physiological functions and human disease. Int J Biochem Cell Biol. 2007;39(1):44-84. doi: 10.1016/j.biocel.2006.07.001. [PubMed:16978905].

12. Benzie IF, Strain JJ. The ferric reducing ability of plasma (FRAP) as a measure of "antioxidant power": the FRAP assay. Anal Biochem. 1996;239(1):70-6. doi:10.1006/abio.1996.0292. [PubMed: 8660627].

13. Hu ML. Measurement of protein thiol groups and glutathione in plasma. Methods Enzymol. 1994;233:380-5. [PubMed: 8015473].

14. Marklund S, Marklund G. Involvement of the superoxide anion radical in the autoxidation of pyrogallol and a convenient assay for superoxide dismutase. Eur JBiochem. 1974;47(3):469-74. [PubMed: 4215654].

15. Aebi H. Catalase in vitro. Methods Enzymol. 1984;105:121-6. [PubMed: 6727660].

16. Paglia DE, Valentine WN. Studies on the quantitative and qualitative characterization of erythrocyte glutathione peroxidase. J Lab Clin Med. 1967;70(1):158-69. [PubMed: 6066618].

17. Ciancarelli I, De Amicis D, Di Massimo C, Carolei A, Ciancarelli MG. Oxidative stress in post-acute ischemic stroke patients after intensive neurorehabilitation. Curr Neurovasc Res. 2012;9(4):266-73. [PubMed: 22873723].

18. Lagowska-Lenard M,Stelmasiak Z, Bartosik-Psujek H. Influence of vitamin C on markers of oxidative stress in the earliest period of ischemic stroke. Pharmacol Rep. 2010;62(4):751-6. [PubMed: 20885017].

19. Sheikh N, Tavilani H, Rezaie A, Vaisi-raygani A, Salimi S. Relationship between estradiol and antioxidant enzymes activity of ischemic stroke. J Biomed Biotechnol. 2009;2009:841468. doi: 10.1155/2009/841468. [PubMed: 19746169].

20. Erel O. A novel automated method to measure total antioxidant response against potent free radical reactions. Clin Biochem. 2004;37(2):112-9. [PubMed: 14725941].

21. Gonullu H, Aslan M, Karadas S, Kati C, Duran L, Milanlioglu A, et al. Serum prolidase enzyme activity and oxidative stress levels in patients with acute hemorrhagic stroke. Scand J Clin Lab Invest. 2014;74(3):199-205. doi:10.3109/00365513.2013.873949. [PubMed: 24456419].

22. Ritzenthaler T, Lhommeau I, Douillard S, Cho TH, Brun J, Patrice T, et al. Dynamics of oxidative stress and urinary excretion of melatonin and its metabolites during acute ischemic stroke. Neurosci Lett. 2013;544:1-4. doi: 10.1016/j.neulet.2013.02.073. [PubMed: 23562888].

23. Parizadeh MR, Azarpazhooh MR, Mobarra N, Nematy M, Alamdari $\mathrm{DH}$, Tavalaie S, et al. Prooxidant-antioxidant balance in stroke patients and 6-month prognosis. Clin Lab. 2011;57(3-4):183-91. [PubMed: 21500725].

24. Tsai NW, Chang YT, Huang CR, Lin YJ, Lin WC, Cheng BC, et al. Association between oxidative stress and outcome in different subtypes of acute ischemic stroke. Biomed Res Int. 2014;2014:256879. doi: 10.1155/2014/256879. [PubMed: 24895559]. 
25. Bektas H, Vural G, Gumusyayla S, Deniz O, Alisik M, Erel O. Dynamic thiol-disulfide homeostasis in acute ischemic stroke patients. Acta Neurol Belg. 2016;116(4):489-94. doi: 10.1007/s13760-016-0598-1. [PubMed: 26782823].

26. Beer C, Blacker D, Hankey GJ, Puddey IB. Association of clinical and aetiologic subtype of acute ischaemic stroke with inflammation, oxidative stress and vascular function: a cross-sectional observational study. Med Sci Monit. 2011;17(9):CR467-73. [PubMed: 21873941].

27. Sanchez-Moreno C, Dashe JF, Scott T, Thaler D, Folstein MF, Martin A. Decreased levels of plasma vitamin $\mathrm{C}$ and increased concentrations of inflammatory and oxidative stress markers after stroke. Stroke. 2004;35(1):163-8. doi: 10.1161/01.STR.0000105391.62306.2E. [PubMed: 14671251].

28. Zhang ZJ. Systematic review on the association between F2isoprostanes and cardiovascular disease. Ann Clin Biochem. 2013;50(Pt 2):108-14. doi: 10.1258/acb.2012.011263. [PubMed: 23019600].

29. Milanlioglu A, Aslan M, Ozkol H, Cilingir V, Nuri Aydin M, Karadas S. Serum antioxidant enzymes activities and oxidative stress levels in patients with acute ischemic stroke: influence on neurological status and outcome. Wien Klin Wochenschr. 2016;128(5-6):169-74. doi: 10.1007/s00508-015-0742-6. [PubMed: 25854910].

30. El Kossi MM, Zakhary MM. Oxidative stress in the context of acute cerebrovascular stroke. Stroke. 2000;31(8):1889-92. [PubMed: 10926952].

31. Demirkaya S, Topcuoglu MA, Aydin A, Ulas UH, Isimer AI, Vural O. Malondialdehyde, glutathione peroxidase and superoxide dismutase in peripheral blood erythrocytes of patients with acute cerebral ischemia. Eur J Neurol. 2001;8(1):43-51. [PubMed: 11509080].

32. Cherubini A, Polidori MC, Bregnocchi M, Pezzuto S, Cecchetti R, Ingegni T, et al. Antioxidant profile and early outcome in stroke patients. Stroke. 2000;31(10):2295-300. [PubMed: 11022053].

33. Aygul R, Kotan D, Demirbas F, Ulvi H, Deniz O. Plasma oxidants and antioxidants in acute ischaemic stroke. J Int Med Res. 2006;34(4):4138. doi: 10.1177/147323000603400411. [PubMed:16989498].

34. Kasner SE, Chalela JA, Luciano JM, Cucchiara BL, Raps EC, McGarvey ML, et al. Reliability and validity of estimating the NIH stroke scale score from medical records. Stroke. 1999;30(8):1534-7. [PubMed: 10436096]. 